\title{
Overcoming Electronic Limits to Optical Phase Measurements with an Optical Phase-only Amplifier
}

\author{
J. Kakande*, R. Slavík, F. Parmigiani, P. Petropoulos and D. J. Richardson \\ Optoelectronics Research Centre, University of Southampton, SO17 IBJ, UK. \\ *jkk@orc.soton.ac.uk
}

\begin{abstract}
We utilize four wave mixing to precisely multiply the modulation depth of a phase encoded signal, demonstrating 3-extra effective bits of resolution at $32 \mathrm{GHz}$. The technique enables a new class of optical signal processing functions.
\end{abstract}

OCIS codes: (060.5060) Phase modulation ;(060.4370) Nonlinear optics, fibers; (190.2620) Harmonic generation and mixing

\section{Introduction}

Photonic signal processing is increasingly finding applications in several fields previously dominated by electronics, including analogue-to-digital conversion (ADC) [1], microwave and terahertz wave generation [2,3], radio-overfiber (ROF) [4], and all-optical regeneration [5]. The benefits of utilizing photonic methods over the electronic equivalent are numerous, including low transmission loss, flat and broadband spectral response, and crucially for ADC applications, lower jitter sampling [1]. In most of these systems, a final optical to electronic conversion (generally incorporating digital sampling) is required. At high speeds, optical amplification is (almost always) utilized to raise the signal power above the optoelectronic receiver's noise floor, hereby maximizing signal-to-noise ratio (SNR). The optical power amplifier has therefore become ubiquitous, with doped fiber amplifiers (DFAs) commonly inserted prior to electro-optic receivers. DFAs by their very nature amplify the signal carrier and information-bearing sidebands equally and as such are very linear. This attribute is desirable when dealing with strong amplitude modulated optical signals. When precise measurement of an analogue signal is required however, such as in a ROF link, it is commonplace to perform only weak modulation in a single dimension such as phase, to ensure that the modulator itself is linear. In that instance, amplification of the signal in a DFA (and indeed in any optical power amplifier) is in fact an impediment, because the energy in the carrier is many orders of magnitude greater than that in the sidebands, and this 'DC' component can saturate, and even damage, the photo-receiver. This leads to a tradeoff between SNR and linearity. In RF, this compromise can be conveniently avoided by using ACcoupled amplifiers that preferentially amplify the sidebands over the redundant carrier. We propose and demonstrate here an optical equivalent of the RF AC-coupled amplifier, obtaining an extra $18 \mathrm{~dB}$ of sideband SNR that in our case led to a 3-bit increase in the effective number of bits (ENOB) for our photo-electric detection system operating at $32 \mathrm{GHz}$. In addition, we highlight how the concept lends itself to a next generation of ultrafast optically assisted ADCs.

\section{Operation Principle and Experimental Set-up}

(a)

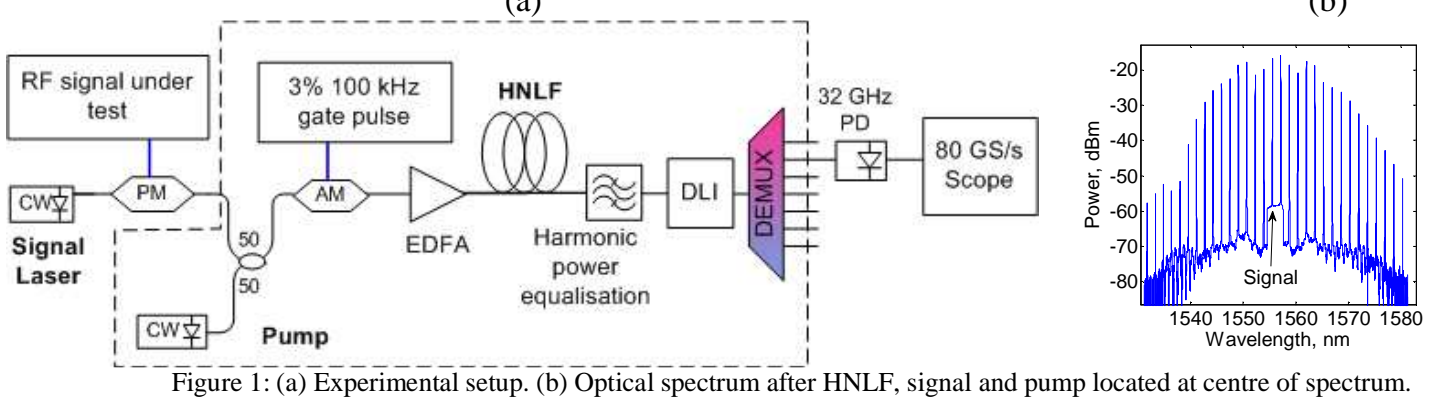

Our device is remarkably simple, and merely requires combining the phase-modulated optical signal with a frequency detuned continuous wave $(\mathrm{CW})$ pump in a highly nonlinear fiber (HNLF). If the dispersion of the HNLF is low, a wideband cascade of four wave mixing (FWM) products is generated [6]. Given that the signal has a constant intensity, it can be written as $\exp (i \phi)$, where $\phi$ is the time varying phase information. Due to the phase matching property of FWM, the $n^{\text {th }}$ harmonic (where $n=1$ corresponds to the signal) can be described as $\exp (\mathrm{in} \phi)$ [6], and the modulation sidebands are enhanced by $n^{2}$ in power relative to those on the signal. In a system in which the limiting factor is the noise of the receiver electronics, and in which conventional optical amplification would only 
serve to saturate the detector (which we demonstrate below), demodulating the $\mathrm{n}^{\text {th }}$ harmonic rather than the signal would allow for $\mathrm{n}$ times better SNR. This holds true when the optical noise floor is lower than the electronic background noise level, which is often the case.

Our proof of principle setup is shown in Fig.1(a). An RF signal was phase modulated onto an optical carrier (laser operating at $1555.8 \mathrm{~nm}$ ) using a $\mathrm{LiNbO}_{3}$ phase modulator. The modulator was driven by various electrical test waveforms generated either from a $24 \mathrm{GS} / \mathrm{s}$ Arbitrary Waveform Generator (AWG) or a fast RF synthesizer. The modulated signal was then combined with the pump $(1557.4 \mathrm{~nm})$. The pump laser was actively controlled to ensure a $200 \mathrm{GHz}( \pm 1 \mathrm{MHz})$ frequency offset between the two waves. The signal and pump were gated at $100 \mathrm{kHz}$ with a $3 \%$ duty cycle before being amplified in an EDFA to increase their peak powers, filtered to suppress out of band amplified spontaneous emission (ASE), and coupled into a the nonlinear stage comprising two HNLFs connected by $12 \mathrm{~m}$ of SMF-28. The total power at the HNLF input was $200 \mathrm{~mW}$. The length, dispersion, dispersion slope, nonlinear coefficient and attenuation of the HNLFs at $1550 \mathrm{~nm}$ were as follows: $500 \mathrm{~m}, 0.06 \mathrm{ps} / \mathrm{nm} / \mathrm{km}$, $0.0035 \mathrm{ps} / \mathrm{nm}^{2} / \mathrm{km}, 18 / \mathrm{W} / \mathrm{km}$ and $1.4 \mathrm{~dB} / \mathrm{km}$ and $500 \mathrm{~m},-0.09 \mathrm{ps} / \mathrm{nm} / \mathrm{km}, 0.016 \mathrm{ps} / \mathrm{nm}^{2} / \mathrm{km}, 11.5 / \mathrm{W} / \mathrm{km}$ and $0.8 \mathrm{~dB} / \mathrm{km}$, respectively. An example of the achieved spectrum is shown in Fig. 1(b), with more detail shown in Fig. 2(a) - the signal was modulation free at the time. The harmonics of interest were then filtered, simultaneously demodulated in a $10 \mathrm{GHz}$ delay line interferometer (DLI) and characterized using a $32 \mathrm{GHz}$ photo-detector and an $80 \mathrm{GS} / \mathrm{s}$ real time oscilloscope (also with $32 \mathrm{GHz}$ bandwidth). The frequency offset stabilization at an integer multiple of the $10 \mathrm{GHz}$ DLI free spectral range (FSR) meant that a single DLI could be used for all harmonics.

(a)

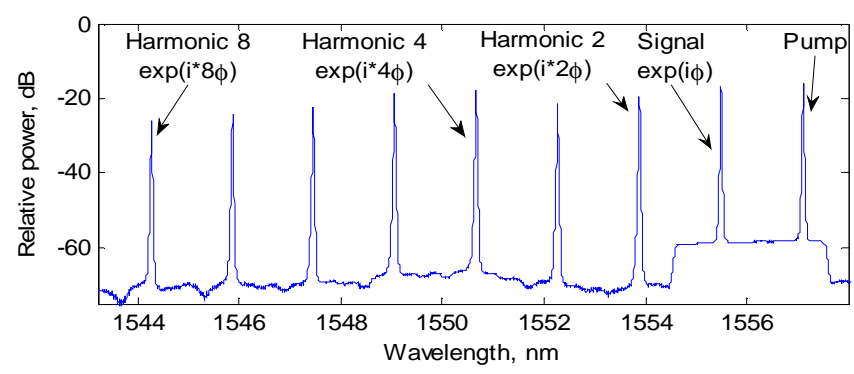

(b)

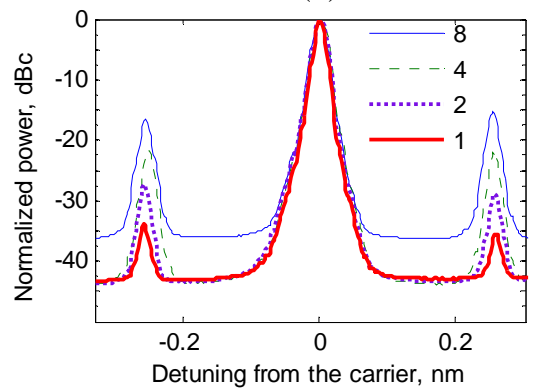

Figure 2: (a) Detail of the output optical spectrum with no phase modulation on signal. (b) Overlaid optical spectra of harmonics 1 (signal), 2, 4 and 8 when the signal was phase modulated at $32 \mathrm{GHz}$. These spectra were captured prior to the DLI, and are normalized to the carrier.

\section{Results}

(a)

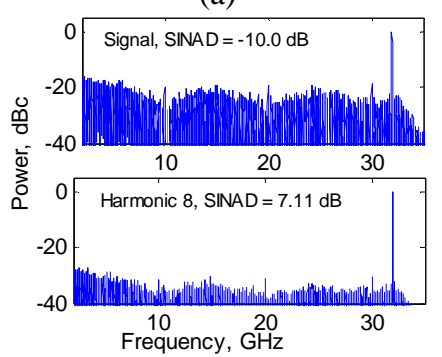

(b)

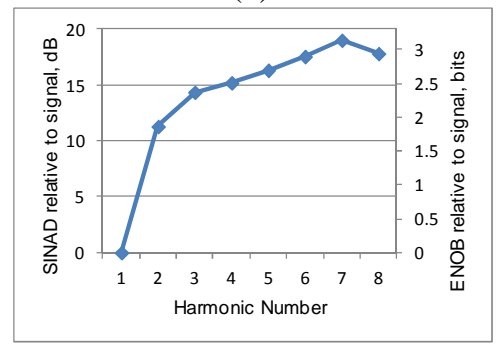

Figure 3: (a) RF spectra of the signal (top) and eighth harmonic (bottom) following detection of the $32 \mathrm{GHz}$ phase modulated signal. (b) SINAD and ENOB of each harmonics 1 (signal) to 8 as calculated from the detected RF data at $32 \mathrm{GHz}$.

Fig. 2(b) shows overlaid optical spectral traces of the signal $(n=1), 2^{\text {nd }}, 4^{\text {th }}$ and $8^{\text {th }}$ harmonics when a $32 \mathrm{GHz} \mathrm{RF}$ sine wave was applied to the modulator. The peak-to-peak voltage was approx $0.03 \mathrm{~V}_{\pi} \cdot$ It is clearly observable that the modulation sideband on the $8^{\text {th }}$ harmonic is increased in power by $18 \mathrm{~dB}$, with the sideband optical signal-tonoise ratio (OSNR) improved by up to $14 \mathrm{~dB}$. The signal and harmonics were then all simultaneously demodulated in the DLI biased at 50\% transmission, before being individually selected, amplified and coupled into the photodetector. Note that the maximum possible signal power was coupled into the detector (as per the detector's specification), therefore further optical power amplification could not have been used to improve the optical-toelectrical conversion. The analog detector output was then captured with the oscilloscope and the data processed offline to calculate standardized figures of merit - signal to noise and distortion ratio (SINAD), as well as the ENOB, calculated from 1-40 GHz [7]. Note that the aggregate noise in the system was dominated by that originating in the receiver electronics due to the weak nature of the phase modulation. The results at $32 \mathrm{GHz}$ are shown in Fig. 
3. The SINAD increased by $18 \mathrm{~dB}$ in going from harmonics 1 to 8 , improving the ENOB by approximately 3 bits. The slight reduction in performance in going from harmonic 7 to 8 is due to the reduced OSNR of the $8^{\text {th }}$ harmonic, Fig. 2(a); this can be easily remedied by increasing the power of the pump to boost the mixing process.

(a)

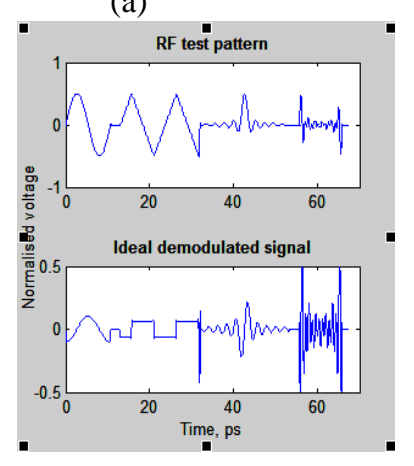

(b)

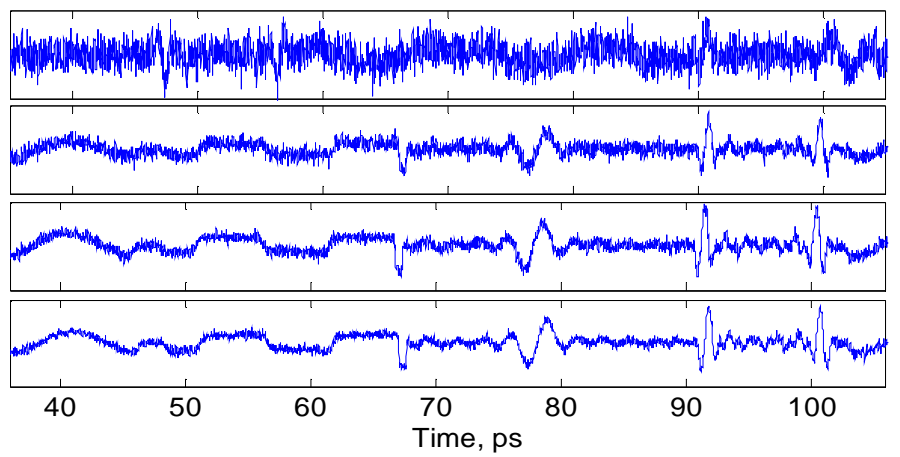

Figure 4: (a) RF test phase pattern, and expected detector output following demodulation in a $10 \mathrm{GHz}$ FSR DLI. (b) Normalised data after demodulating signal, harmonic 2,4 and 8 (from top to bottom respectively).

Finally, we show the system operating with a wideband input RF signal occupying $10 \mathrm{GHz}$. The chosen electrical pattern can be seen in Fig. 4 (a, top), and the ideal demodulated output (modeled) is shown in Fig. 4 (a, bottom). The various harmonics (1, 2, 4 and 8) after detection are shown in Fig. 4(b) with their powers normalized. The improvement in going from the signal ( $4 \mathrm{~b}$, top) to the $8^{\text {th }}$ harmonic is clearly visible. Calculating the ENOB in this instance is not directly possible due to the complex nature of the demodulating function.

\section{Discussion and Conclusion}

It is important to focus on the fact that this improvement is achieved by simply combining the signal with a CW laser in an optical fiber. In fact, the optical fiber could be integrated as part of the transmission channel, meaning zero additional latency. In contrast, other ENOB enhancement techniques, such as RF clock jitter reduction, require either an extremely low noise RF clock source and specially designed electronics, or the use of a mode-locked laser to perform pre-sampling [1]. Both these options will have significant cost and power implications. In addition, by either using a pulsed source as the pump, or optically sampling the harmonics after the FWM, the benefits of low jitter sampling can be combined with the significant power of phase amplification. Furthermore, the concept provides a route to building next generation optically assisted ADCs. Because the harmonics are precise integer multiples of the original channel, the device can be thought of as a modulo- $2 \pi$ calculator. Consequently, detecting each of the harmonics $1,2,4,8 \ldots$, using single bit electronic receivers, directly allows one to build ultra-high speed ADCs; at the moment this very modulo arithmetic is internally performed within the electronic ADC after detection.

In conclusion, we have used a cascaded FWM mixing process to generate the integer phase harmonics of a signal under test, and utilized this to achieve SNR enhancement without needing to deploy traditional optical amplification. This technique allowed us to improve the signal-to-noise-and-distortion-ratio performance of an optical-to-electrical converter by $18 \mathrm{~dB}$, leading to 3 extra effective bits in detection. This technique will find many applications in photonic systems relying on optical phase to carry information, as well as future optically assisted ADCs.

\section{Acknowledgement}

This work is supported by the EPSRC grant EP/I01196X: Transforming the Future Internet: The Photonics Hyperhighway.

\section{References}

[1] A. Khilo et al, "Photonic ADC: overcoming the bottleneck of electronic jitter," Opt. Express 20, 4454-4469 (2012).

[2] A.J. Seeds and K.J. Williams, "Microwave Photonics," J. Lightwave Technol. 24, 4628-4641 (2006).

[3] S. Fukushima, C. F. C. Silva, Y. Muramoto, and A. J. Seeds, "Optoelectronic millimeter-wave synthesis using an optical frequency comb generator, optically injection locked lasers, and a unitraveling-carrier photodiode," J. Lightwave. Technol. 21, 3043-3051 (2003).

[4] Y. Kim, S. Doucet, M. E. Mousa Pasandi, and S. LaRochelle, "Optical multicarrier generator for radio-over-fiber systems," Opt. Express 16, 1068-1076 (2008).

[5] K. Croussore, I. Kim, Ch. Kim, Y. Han, and G. Li, G. Phase-and-amplitude regeneration of differential phase-shift keyed signals using a phase-sensitive amplifier," Opt. Express 14, 2085-2094 (2006).

[6] J Kakande, et. al., "Multilevel quantization of optical phase in a novel coherent parametric mixer architecture," Nat Photon 5, 12, pp 748-752 (2011).

[7] "IEEE Standard for Terminology and Test Methods for Analog-To-Digital Converters," IEEE Std 1241-2000 ( 2001). 